\title{
An Efficient Polynomial Time Algorithm for a Class of Generalized Linear Multiplicative Programs with Positive Exponents
}

\author{
Bo Zhang, ${ }^{1}$ YueLin Gao $\mathbb{D}^{2,3}$ Xia Liu, ${ }^{1}$ and XiaoLi Huang ${ }^{3}$ \\ ${ }^{1}$ School of Mathematics and Statistics, Ningxia University, Yinchuan 750021, China \\ ${ }^{2}$ Ningxia Province Key Laboratory of Intelligent Information and Data Processing, North Minzu University, \\ Yinchuan 750021, China \\ ${ }^{3}$ School of Mathematics and Information Science, North Minzu University, Yinchuan 750021, China \\ Correspondence should be addressed to YueLin Gao; gaoyuelin@263.net
}

Received 23 September 2020; Accepted 6 February 2021; Published 26 February 2021

Academic Editor: Guoqiang Wang

Copyright (c) 2021 Bo Zhang et al. This is an open access article distributed under the Creative Commons Attribution License, which permits unrestricted use, distribution, and reproduction in any medium, provided the original work is properly cited.

\begin{abstract}
This paper explains a region-division-linearization algorithm for solving a class of generalized linear multiplicative programs (GLMPs) with positive exponent. In this algorithm, the original nonconvex problem GLMP is transformed into a series of linear programming problems by dividing the outer space of the problem GLMP into finite polynomial rectangles. A new two-stage acceleration technique is put in place to improve the computational efficiency of the algorithm, which removes part of the region of the optimal solution without problems GLMP in outer space. In addition, the global convergence of the algorithm is discussed, and the computational complexity of the algorithm is investigated. It demonstrates that the algorithm is a complete polynomial time approximation scheme. Finally, the numerical results show that the algorithm is effective and feasible.
\end{abstract}

\section{Introduction}

Consider a class of generalized linear multiplicative programs (GLMPs):

$$
(\mathrm{LFP}):\left\{\begin{array}{l}
\min \quad f(x)=\prod_{i=1}^{p}\left(c_{i}^{T} x+d_{i}\right)^{\alpha_{i}} \\
\text { s.t. } x \in X=\left\{x \in \mathbb{R}^{n} \mid A x \leq b, x \geq 0\right\} .
\end{array}\right.
$$

Here, $p \geq 2, X$ is a nonempty bounded closed set, $A \in \mathbb{R}^{m \times n}, b \in \mathbb{R}^{m}, c_{i} \in \mathbb{R}^{n}, d_{i} \in \mathbb{R}$, and $\alpha_{i}>0$. T represents the transpose of a vector (e.g., $c_{i}^{T}$ represents the transpose of a vector $c_{i}$ ). Besides, we assume that for any $x \in \mathrm{X}$, all make $c_{i}^{T} x+d_{i}>0, i=1,2, \ldots, p$.

The problem GLMP usually has multiple nonglobal local optimal solutions and is a class of NP-hard problems [1], which can be widely used in the fields of finance optimization [2,3], robust optimization [4], microeconomics [5], and multiobjective decision making $[6,7]$. In addition, the GLMP also includes a wide range of mathematical programming categories, such as linear multiplicative programming, quadratic programming, bilinear programming, and so on. Therefore, for these and various other reasons, GLMP has caught the attention of many experts, scholars, and engineering practitioners who have studied this theory and set off a new wave of global optimization learning. With the increasing dependence of practical problems on modeling optimization, local optimization theory and global optimization algorithms have made remarkable progress. However, compared with local optimization algorithm, the theory of global optimization algorithm is still quite insufficient. There are many methods to study this kind of problems, such as level set algorithm [8], heuristic algorithm $[9,10]$, branch and bound algorithm [11-13], outer approximation algorithm [14], parametric simplex algorithm [15], and so on, but these methods do not give the computational complexity of the algorithm. In addition, Depetrini and Locatelli [16] considered the problem of minimizing the product of two affine functions over a polyhedron set and proposed a polynomial time approximation algorithm. Locatelli [17] presented an approximate algorithm for solving more general types of global 
optimization problems and deduced the computational complexity of the algorithm, but the numerical results of the algorithm are lacking. Recently, Shen and Wang [18] also proposed a full polynomial time approximation algorithm for resolving the problem GLMP globally, but there is no acceleration technique. Moreover, for a more comprehensive overview of the GLMP, we encourage the readers to go through the more detailed literature [8, 19-21].

In this paper, in order to solve the GLMP, two approximation algorithms are proposed, which is mainly by establishing a nonuniform grid; the process of solving the original problem is transformed into the process of solving a series of linear problems; it is proved that the proposed algorithm can obtain a global $\mathcal{\varepsilon}$-approximation solution for GLMP. Besides, we put forward a two-stage acceleration technique to speed up Algorithm 1, which yields Algorithm 2. Then, by discussing the computational complexity of the algorithm, it is shown that the two algorithms are polynomial time approximation algorithms. Numerical experiments show that the performance of Algorithm 2 is obviously better than that of Algorithm 1, and the numerical results in Table 2 show that in solving problem 1-3, Algorithm 2 uses less CPU running time and iterations than $[17,18]$.

The rest of this paper is organized as follows. In Section 2, we first transform the problem GLMP into its equivalent optimization problem EOP and give its region-decomposition-linearization technique. Section 3 presents the global $\mathcal{E}$-approximation algorithm for problem GLMP and obtains the convergence for the proposed algorithm. In Section 4, we give the computational complexity for the proposed algorithm and carry out some numerical experiments in Section 5 to verify the feasibility and effectiveness of the algorithm. The concluding section is a simple summary.

\section{Equivalence Problem and Its Linearization Technique}

In this section, we will give the equivalent optimization problem EOP of the problem GLMP, then give the corresponding properties by studying the objective function of the EOP, and then explain the linearization technique of the equivalent problem.

2.1. Equivalent Problems and Their Properties. In order to solve the problem GLMP, the definition of global $\epsilon$-approximation solution is given below.

Definition 1. Let $x^{*}$ be a global optimal solution to the problem GLMP at a given precision $\varepsilon \in(0,1)$. If $\hat{x} \in X$ satisfies $f(\hat{x}) \leq(1+\varepsilon) f\left(x^{*}\right), \widehat{x}$ is referred to as the global approximation of the problem GLMP.

To obtain the global $\varepsilon$-approximation solution for GLMP, let $f_{i}(x)=c_{i}^{T} x+d_{i}, l_{i}=\min _{x \in X} f_{i}(x)$.

Theorem 1. For each $i=1,2, \ldots, p$, let $\tilde{x}^{i}=\operatorname{argmin}_{x \in X} f_{i}(x), \quad Q=\cup_{i=1}^{p} \tilde{x}^{i}, \quad \check{x}=\operatorname{argmin}_{x \in Q} f(x)$, $\widetilde{U}=f(\check{x})$. Then, for each $i \in\{1,2, \ldots, p\}$, let $M_{i}=\prod_{j=1, j \neq i}^{p} l_{j}^{\alpha_{j}}$; then, $f_{i}\left(x^{*}\right) \leq u_{i}$ with $u_{i}=\left(\tilde{U} / M_{i}\right)^{\left(1 / \alpha_{i}\right)}$.
Proof. It is easy to know that for any $i \in\{1,2, \ldots, p\}$, there are $l_{i} \leq f_{i}\left(x^{*}\right)$; thus,

$$
\prod_{j=1, j \neq i}^{p} l_{j}^{\alpha_{j}}\left(f_{i}\left(x^{*}\right)\right)^{\alpha_{i}} \leq \prod_{i=1}^{p}\left(f_{i}\left(x^{*}\right)\right)^{\alpha_{i}}=f\left(x^{*}\right) \leq f(\check{x})=\tilde{U} .
$$

Therefore, $f_{i}\left(x^{*}\right) \leq\left(\widetilde{U} / M_{i}\right)^{\left(1 / \alpha_{i}\right)}=u_{i}$ and then the conclusion holds.

Next, according to Theorem 1 , for each $i=1,2, \ldots, p$, $u_{i}=\left(\tilde{U} / M_{i}\right)^{\left(1 / \alpha_{i}\right)}$ provide an upper bound for every $f_{i}\left(x^{*}\right)$.

On the basis of the above definition of $l_{i}$ and $u_{i}$, define the rectangle $H$ as follows.

$$
H=\left[l_{1}, u_{1}\right] \times\left[l_{2}, u_{2}\right] \times \cdots \times\left[l_{p}, u_{p}\right] .
$$

Moreover, the rectangle $H$ is also called the outer space of the GLMP. Thus, by introducing variable $y=\left(y_{1}, y_{2}, \ldots, y_{p}\right)^{T} \in H$, the problem GLMP is equivalent to the following problem $\mathrm{P} 1$.

$$
(\mathrm{P} 1)\left\{\begin{array}{l}
\min \quad h(y)=\prod_{i=1}^{p} y_{i}^{\alpha_{i}}, \\
\begin{array}{l}
f_{i}(x) \leq y_{i}, i=1,2, \ldots, p \\
x \in X, y \in H .
\end{array}
\end{array}\right.
$$

Next, the equivalence of problems GLMP and P1 is explained by Theorem 1 .

Theorem 2. $x^{*}$ is a global optimal solution of problem GLMP if and only if $\left(x^{*}, y^{*}\right)$ is an optimal solution of problem $P 1$ and $y_{i}^{*}=f_{i}\left(x^{*}\right), i=1,2, \ldots, p$.

Proof. Let $y_{i}^{*}=f_{i}\left(x^{*}\right), i=1,2, \ldots, p$ if $x^{*}$ is a global optimal solution of the problem GLMP. Then, then it is obvious that $\left(x^{*}, y^{*}\right)$ is a feasible solution to P1. Suppose $\left(x^{*}, y^{*}\right)$ is not an optimal solution of P1; then, there is at least one feasible solution $(\bar{x}, \bar{y})$ of $\mathrm{P} 1$, which makes

$$
f(\bar{x})=\prod_{i=1}^{p}\left(f_{i}(\bar{x})\right)^{\alpha_{i}} \leq \prod_{i=1}^{p} \bar{y}_{i}^{\alpha_{i}}<\prod_{i=1}^{p}\left(y_{i}^{*}\right)^{\alpha_{i}}=\prod_{i=1}^{p}\left(f_{i}\left(x^{*}\right)\right)^{\alpha_{i}}=f\left(x^{*}\right),
$$

which contradicts the optimality of the $x^{*}$, so the hypothesis does not hold, and then $\left(x^{*}, y^{*}\right)$ is an optimal solution of P1.

Conversely, if $\left(x^{*}, y^{*}\right)$ is an optimal solution for P1 and if there is a $i \in\{1,2, \ldots, p\}$ that makes $f_{i}\left(x^{*}\right)<y_{i}^{*}$, let $\tilde{y}_{i}=f_{i}\left(x^{*}\right)$, then $\left(x^{*}, \tilde{y}\right)$ is a feasible solution for P1 and

$$
\prod_{i=1}^{p} \tilde{y}_{i}^{\alpha_{i}}<\prod_{i=1}^{p}\left(y_{i}^{*}\right)^{\alpha_{i}}
$$

which contradicts the optimality of $\left(x^{*}, y^{*}\right)$, so $y_{i}^{*}=f_{i}\left(x^{*}\right), i=1,2, \ldots, p$. Suppose $x^{*}$ is not a global optimal solution of the problem GLMP; then, there must be a $\bar{x} \in X$ that makes $f(\bar{x})<f\left(x^{*}\right)$. Let $\bar{y}_{i}=f_{i}(\bar{x})$; obviously, $(\bar{x}, \bar{y})$ is a feasible solution to $\mathrm{P} 1$, so we have 
(1) Step 0 (initialization). Set $\varepsilon \in(0,1), \delta=(1+\varepsilon)^{(1 / \rho)}, F=+\infty, k=0$. By using formulas (22) and (23), the ratio used for the two consecutive segments in each dimension is $\delta$, which subdivides $H$ into smaller rectangles. Represent the vertex of each small rectangle as $\nu=\left(v_{1}, v_{2}, \ldots, v_{p}\right)$, which is stored in the set $B^{\delta}$.

(2) Step 1. Select a point $v$ from the $B^{\delta}$, solve the linear programming problem $\left(\mathrm{LP}_{v}\right)$, and let $B^{\delta}=B^{\delta} \backslash \nu$.

(3) Step 2. If the problem $\left(\operatorname{LP}_{v}\right)$ is solvable, then $D(\nu) \neq \varnothing$, and let $g(v)=\prod_{i=1}^{p}\left(v_{i}\right)^{\alpha_{i}}$; if $g(v)<F$, let $F=g(\nu), \bar{v}=\nu, x_{\bar{v}}=x_{v}$; if $B^{\delta} \neq \varnothing$, set $k=k+1$ and go to Step 1; otherwise, the algorithm terminates; let

Algorithm 1: Original algorithm.

$$
\prod_{i=1}^{p}\left(\bar{y}_{i}\right)^{\alpha_{i}}=f(\bar{x})<f\left(x^{*}\right)=\prod_{i=1}^{p}\left(y_{i}^{*}\right)^{\alpha_{i}}
$$

which contradicts the optimality of $\left(x^{*}, y^{*}\right)$. Therefore, $x^{*}$ is the global optimal solution of the problem GLMP, which proves to be completed.

It is easy to understand from Theorem 2 that the problems GLMP and P1 are equivalent and have the same global optimal value.

Then, for a given $y \in H$, define the set

$$
D(y)=\left\{x \in X \mid f_{i}(x) \leq y_{i}, i=1,2, \ldots, p\right\},
$$

and function

$$
g(y)= \begin{cases}h(y), & D(y) \neq \varnothing, \\ +\infty, & D(y)=\varnothing .\end{cases}
$$

Then, the problem P1 is equivalent to the following equivalent optimization problem.

$$
\text { (EOP) } \begin{cases}\min & g(y) \\ \text { s.t. } & y \in H .\end{cases}
$$

Theorem 3. $y^{*}$ is the global optimal solution of the problem EOP if and only if $\left(x^{*}, y^{*}\right)$ is the optimal solution of $P 1$ and $y_{i}^{*}=f_{i}\left(x^{*}\right), i=1,2, \ldots, p$.

Proof. Suppose $\left(x^{*}, y^{*}\right)$ is an optimal solution of $\mathrm{P} 1$; then, according to Theorem 2, we can know $y_{i}^{*}=f_{i}\left(x^{*}\right), i=1,2, \ldots, p$ and $y^{*} \in H$. In addition, $h\left(y^{*}\right)=g\left(y^{*}\right)=\prod_{i=1}^{p}\left(y_{i}^{*}\right)^{\alpha_{i}}$. Suppose that $y^{*}$ is not the global optimal solution of the problem EOP; there must be a $\bar{y} \in H$ such that $g(\bar{y})<g\left(y^{*}\right)$ and $D(\bar{y}) \neq \varnothing$; then, there must also be a $\bar{x} \in D(\bar{y})$ such that $f_{i}(\bar{x}) \leq \bar{y}_{i}, i=1,2, \ldots, p$. Then, $(\bar{x}, \bar{y})$ is a feasible solution of $\mathrm{P} 1$; there is $h(\bar{y})=g(\bar{y})<g\left(y^{*}\right)=h\left(y^{*}\right)$, which contradicts the optimality of $\left(x^{*}, y^{*}\right)$, so the hypothesis does not hold, so $y^{*}$ is the global optimal solution of the problem.

On the other hand, if $y^{*}$ is a global optimal solution of the problem EOP, then $D\left(y^{*}\right) \neq \varnothing$, and there must be a $x^{*} \in D\left(y^{*}\right)$ such that $\left(x^{*}, y^{*}\right)$ is a feasible solution of $\mathrm{P} 1$. Suppose $\left(x^{*}, y^{*}\right)$ is not the global optimal solution of the problem P1; then, there must be an optimal solution $(\bar{x}, \bar{y})$ to the problem P1 such that $h(\bar{y})<h\left(y^{*}\right), \bar{y}_{i}=f_{i}(\bar{x}), i=1,2, \ldots, p$, so $D(\bar{y}) \neq \varnothing$ and $g(\bar{y})=h(\bar{y})<h\left(y^{*}\right)=g\left(y^{*}\right)$, which contradicts the fact that $y^{*}$ is the global optimal solution of the problem EOP.
Therefore, $\left(x^{*}, y^{*}\right)$ is the global optimal solution of $\mathrm{P} 1$, and $y_{i}^{*}=f_{i}\left(x^{*}\right), i=1,2, \ldots, p$ can be obtained from Theorem 2 and then proved to be over.

Through Theorem 3, the problems EOP and P1 have the same global optimal value, so combined with Theorem 2 , the problems EOP and GLMP are also equivalent. Therefore, we can solve the equivalent problem EOP instead of addressing the problem GLMP.

Next, we consider the following linear programming problem:

$$
\operatorname{LP}_{y}\left\{\begin{array}{l}
\min \sum_{i=1}^{p} \frac{\alpha_{i} f_{i}(x)}{y_{i}} \\
\text { s.t. } x \in D(y) .
\end{array}\right.
$$

If $D(y) \neq \varnothing$, the optimal solution to the problem $\mathrm{LP}_{y}$ is recorded as $x_{y}$, and let $\tilde{y}_{i}=f_{i}\left(x_{y}\right), \rho=\sum_{i=1}^{p} \alpha_{i}>0$; then,

$$
\rho=\sum_{i=1}^{p} \frac{\alpha_{i} y_{i}}{y_{i}} \geq \sum_{i=1}^{p} \frac{\alpha_{i} f_{i}(x)}{y_{i}}, \quad \forall x \in D(y) .
$$

Furthermore, according to the Jensen inequality, we have

$$
\sum_{i=1}^{p} \frac{\alpha_{i} f_{i}\left(x_{y}\right)}{y_{i}} \geq \rho\left(\prod_{i=1}^{p}\left(\frac{f_{i}\left(x_{y}\right)}{y_{i}}\right)^{\alpha_{i}}\right)^{(1 / \rho)}=\rho\left(\frac{g(\tilde{y})}{g(y)}\right)^{(1 / \rho)}
$$

and then

$$
\rho \geq \rho\left(\frac{g(\widetilde{y})}{g(y)}\right)^{(1 / \rho)}, \quad g(\widetilde{y}) \leq g(y) .
$$

Theorem 4. Suppose $x^{*} \in X$ is a global optimal solution of the original problem GLMP; let $y_{i}^{*}=f_{i}\left(x^{*}\right), i=1,2, \ldots, p$; then, $y^{*}=\left(y_{1}^{*}, y_{2}^{*}, \ldots, y_{p}^{*}\right)^{T} \in H$ and $x^{*}$ is also a global optimal solution of the problem $\left(L P_{y^{*}}\right)$.

Proof. Firstly, according to Theorems 2 and 3, we know that $y^{*}$ is a global optimal solution of the problem EOP. Then, by using formula (14) and the optimality of the global optimal solution $y^{*}$ of the EOP, we can see that $x^{*}$ is an optimal solution of the problem $\left(L P_{y^{*}}\right)$.

Next, the properties of the function $g(y)$ over $H$ are given by Theorem 5 .

Theorem 5. For a given precision $\varepsilon \in(0,1)$, let $\delta=(1+\varepsilon)^{(1 / \rho)}$; then, for any $\bar{y} \in H$, there is 


$$
g(\bar{y}) \leq(1+\varepsilon) g(y), \quad \forall y \in\left[\frac{\bar{y}}{\delta}, \bar{y}\right]
$$

In addition, if $D(\bar{y}) \neq \varnothing$, the optimal solution to the problem $\left(\mathrm{LP}_{\bar{y}}\right)$ is recorded as $\bar{x}$; then, let $\widetilde{y}_{i}=f_{i}(\bar{x})(i=1,2, \ldots, p)$; there is also

$$
g(\widetilde{y}) \leq g(\bar{y}) \leq(1+\varepsilon) g(y), \quad \forall y \in\left[\frac{\bar{y}}{\delta}, \bar{y}\right] .
$$

Proof. For all $\bar{y} \in H$, according to the definition of $D(y)$ and $\delta=(1+\varepsilon)^{(1 / \rho)}>1$, one can know $D(\bar{y} / \delta) \subseteq D(\bar{y})$.

If $D(\bar{y} / \delta) \neq \varnothing$, for any $y \in[(\bar{y} / \delta), \bar{y}]$, we have $D(y) \neq \varnothing$; obviously, $\quad g(\tilde{y}) \leq g(\bar{y})$ and $y_{i} \geq\left(\bar{y}_{i} / \delta\right)$ for each $i=1,2, \ldots, p$. Thus,

$$
\prod_{i=1}^{p}\left(\frac{\bar{y}_{i}}{\delta}\right)^{\alpha_{i}} \leq \prod_{i=1}^{p} y_{i}^{\alpha_{i}}
$$

Moreover, according to the definition of function $g(y)$, $g(y)=\prod_{i=1}^{p} y_{i}^{\alpha_{i}}$; thus,

$$
g\left(\frac{\bar{y}}{\delta}\right)=\prod_{i=1}^{p}\left(\frac{\bar{y}_{i}}{\delta}\right)^{\alpha_{i}}=\frac{1}{\delta^{\rho}} \prod_{i=1}^{p} \bar{y}_{i}^{\alpha_{i}}=\frac{1}{\delta^{\rho}} g(\bar{y}) .
$$
have

And in combination with the formulas (17) and (18), we

$$
g(y) \geq g\left(\frac{\bar{y}}{\delta}\right)=\frac{1}{\delta^{\rho}} g(\bar{y}), \quad \forall y \in\left[\frac{\bar{y}}{\delta}, \bar{y}\right] .
$$

Further, through formula (19) and combined with the definition of $\delta$, we can understand that formula (16) is formed, and formula (15) is of course also true.

If $D(\bar{y} / \delta)=\varnothing, D(\bar{y}) \neq \varnothing$, it is clear that the inequality $g(\widetilde{y}) \leq g(\bar{y})$ is established.

For all $y \in[(\bar{y} / \delta), \bar{y}]$, if $D(y) \neq \varnothing$, we have $y_{i} \geq\left(\bar{y}_{i} / \delta\right)(i=1,2, \ldots, p)$, and $y \neq(\bar{y} / \delta)$; then,

$$
\prod_{i=1}^{p}\left(\frac{\bar{y}_{i}}{\delta}\right)^{\alpha_{i}} \leq g(y)=\prod_{i=1}^{p} y_{i}^{\alpha_{i}}
$$

Besides,

$$
g(\bar{y})=\prod_{i=1}^{p} \bar{y}_{i}^{\alpha_{i}}=\delta^{\rho} \prod_{i=1}^{p}\left(\frac{\bar{y}_{i}}{\delta}\right)^{\alpha_{i}} .
$$

By using the definition of $\delta$ and formulas (20) and (21), one can infer that formulas (15) and (16) hold.

If $D(y)=\varnothing$ and $g(y)=+\infty$, then formulas (15) and (16) obviously hold.

If $D(\bar{y})=\varnothing$, the problem $\left(\mathrm{LP}_{\bar{y}}\right)$ is not solved, and for any $y \in[(\bar{y} / \delta), \bar{y}]$, there is $D(y)=\varnothing$, then $g(y)=+\infty$, so formula (15) is clearly established and the proof of the conclusion is completed.

Theorem 5 shows that for any $\bar{y} \in H$, we can determine whether the $D(\bar{y})$ is not empty by solving the linear programming problem $\left(\mathrm{LP}_{\bar{y}}\right)$ and then determine whether formula (16) holds.
2.2. Linearization Techniques. The objective function of the problem EOP is still nonconvex compared to the problem GLMP. But the space $H$ in which the variable $y$ of the objective function is located is $p$ dimensions. Therefore, based on the above discussion, in order to solve the EOP, for a given $\varepsilon \in(0,1)$, we first split the outer space $H$ on each dimension at a ratio of $\delta=(1+\varepsilon)^{(1 / \rho)}$, thus producing several small rectangles.

To do this, let

$$
\gamma_{i}=\arg \max \left\{\sigma \in \mathbb{N} \mid l_{i} \delta^{\sigma} \leq u_{i}\right\}, \quad i=1,2, \ldots, p,
$$

where $\mathbb{N}$ represents a non-negative integer set. Therefore, the number of these small rectangles is finite, and the set of all their vertices is

$$
B^{\delta}=\left\{v_{1}, v_{2}, \ldots, v_{p} \mid v_{i} \in P_{i}^{\delta}, i=1,2, \ldots, p\right\},
$$

where $P_{i}^{\delta}=\left\{l_{i}, l_{i} \delta, \ldots, l_{i} \delta^{\gamma_{i}}\right\}$. Obviously, for each $y \in H$, there must be a vertex $\left(\nu_{1}, v_{2}, \ldots, v_{p}\right) \in B^{\delta}$ making $y_{i} \in\left[\nu_{i}, \delta v_{i}\right], i=1,2, \ldots, p$. Then, it can be concluded that the rectangle $H$ can be approximated by the set $B^{\delta}$.

Next, by using the set $B^{\delta}$, the process of solving the problem EOP can be transformed into solving a series of subproblems. To this end, for each $v \in B^{\delta}$, we need to consider the value of the $g(\nu)$, that is, we need to determine whether the set $D(\nu)$ is not empty. According to Theorem 5, we can determine whether $D(\nu)$ is not empty by solving the linear programming problem $\left(\mathrm{LP}_{\nu}\right)$. Therefore, for each vertex $v \in B^{\delta}$, the following linear programming subproblem needs to be solved here, that is,

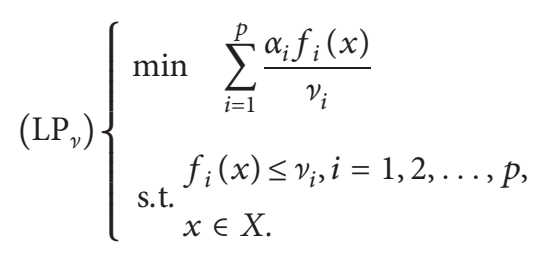

On the basis of the conclusion of Theorem 5 , if the problem $\left(\mathrm{LP}_{v}\right)$ can be solved (its solution is recorded as $x_{v}$ ), then

$$
\widetilde{v}=\left(f_{1}\left(x_{v}\right), f_{2}\left(x_{v}\right), \ldots, f_{p}\left(x_{v}\right)\right)^{T} \in H
$$

and thus

$$
g(\widetilde{\nu}) \leq g(\nu) \leq(1+\varepsilon) g(y), \quad \forall y \in\left[\frac{\nu}{\delta}, \nu\right] .
$$

\section{Analysis of Algorithm and Its Computational Complexity}

This section brings an approximate algorithm based on linearization-decomposition to solve the problem EOP. After that, the analysis of its computational complexity is proved accordingly.

3.1. Approximate Algorithm. To solve the EOP, we subdivide the external space $H$ into a finite number of small rectangles with ratio $\delta$ and put all the vertices of these small rectangles into the set $B^{\delta}$. 
Then, for each vertex $v \in B^{\delta}$, by solving the linear programming problem $\left(\mathrm{LP}_{\gamma}\right)$, if $\left(\mathrm{LP}_{\gamma}\right)$ is feasible and has an optimal solution $x_{v}$, then $D(\nu) \neq \varnothing$, and we can obtain a feasible solution $\widetilde{\nu}$ (formula (25)) of the EOP according to $x_{v}$, which makes

$$
g(\widetilde{\nu}) \leq g(\nu) \leq(1+\varepsilon) g(y), \quad \forall y \in\left[\frac{\nu}{\delta}, \nu\right] .
$$

If there is a $\widetilde{v}$ that satisfies $g(\widetilde{v}) \leq(1+\varepsilon) g\left(y^{*}\right)$, then

$$
f\left(x_{v}\right)=\prod_{i=1}^{p}\left(f_{i}\left(x_{v}\right)\right)^{\alpha_{i}}=\prod_{i=1}^{p} \widetilde{v}_{i}^{\alpha_{i}}=g(\widetilde{\nu}) \leq(1+\varepsilon) g\left(y^{*}\right)=(1+\varepsilon) f\left(x^{*}\right),
$$

and thus $x_{v}$ is a global $\varepsilon$-approximation solution of the problem GLMP. The specific algorithm steps are as follows.

(1) Step 0 (initialization). Set $\varepsilon \in(0,1), \delta=(1+\varepsilon)^{(1 / \rho)}, F=+\infty, k=0$. By using formulas (22) and (23), the ratio used for the two consecutive segments in each dimension is $\delta$, which subdivides $H$ into smaller rectangles. Represent the vertex of each small rectangle as $v=\left(\nu_{1}, v_{2}, \ldots, v_{p}\right)$, which is stored in the set $B^{\delta}$.

(2) Step 1. Select a point $v$ from the $B^{\delta}$, solve the linear programming problem $\left(\mathrm{LP}_{\nu}\right)$, and let $B^{\delta}=B^{\delta} \backslash \nu$.

(3) Step 2. If the problem $\left(\mathrm{LP}_{\gamma}\right)$ is solvable, then $D(\nu) \neq \varnothing$, and let $g(\nu)=\prod_{i=1}^{p}\left(v_{i}\right)^{\alpha_{i}}$; if $g(\nu)<F$, let $F=g(\nu), \bar{v}=\nu, x_{\bar{v}}=x_{\gamma}$; if $B^{\delta} \neq \varnothing$, set $k=k+1$ and go to Step 1; otherwise, the algorithm terminates; let

$$
\widetilde{v}_{i}=f_{i}\left(x_{\bar{v}}\right), \quad i=1,2, \ldots, p, \widetilde{v}=\left(\widetilde{v}_{1}, \widetilde{v}_{2}, \ldots, \widetilde{v}_{p}\right)^{T}
$$

and then $x_{\bar{v}}, \widetilde{v}$ is a global $\mathcal{E}$-approximation solution to problems GLMP and EOP, respectively.

Theorem 6. For a given precision $\varepsilon \in(0,1)$, let $\delta=(1+\varepsilon)^{(1 / \rho)}, \quad \bar{v}=\arg \min \left\{g(\nu) \mid \nu \in B^{\delta}\right\}$, and $x_{\bar{\nu}}$ be an optimal solution of the linear programming problem $\left(L P_{\bar{\nu}}\right)$. Then, Algorithm 1 will get a global $\varepsilon$-approximation solution $x_{\bar{v}}$ for problem GLMP, i.e.,

$$
f\left(x_{\bar{v}}\right) \leq(1+\varepsilon) f\left(x^{*}\right),
$$

where $x^{*}$ is the global optimal solution to the original problem GLMP.

Proof. Let

$$
y_{i}^{*}=f_{i}\left(x^{*}\right), \quad i=1,2, \ldots, p .
$$

According to Theorem 1, we have

$$
l_{i} \leq y_{i}^{*} \leq u_{i}, \quad i=1,2, \ldots, p .
$$

Then, formula (32) implies that $y^{*}=\left(y_{1}^{*}, y_{2}^{*}, \ldots, y_{p}^{*}\right)^{T} \in H$, so there must be a $v^{*} \in B^{\delta}$ which makes

$$
\frac{v_{i}^{*}}{\delta} \leq y_{i}^{*} \leq v_{i}^{*}, \quad i=1,2, \ldots, p .
$$

So, using Theorem 5 on the small rectangle $\left[\left(\nu^{*} / \delta\right), v^{*}\right]$, there will be

$$
f\left(x^{*}\right)=\prod_{i=1}^{p}\left(y_{i}^{*}\right)^{\alpha_{i}}=g\left(y^{*}\right) \geq \prod_{i=1}^{p}\left(\frac{v_{i}^{*}}{\delta}\right)^{\alpha_{i}}=\left(\frac{1}{\delta}\right)^{\Sigma_{i=1}^{p} \alpha_{i}} \prod_{i=1}^{p}\left(v_{i}^{*}\right)^{\alpha_{i}}=\frac{1}{\delta^{\rho}} g\left(v^{*}\right) .
$$

Thus,

$$
\delta^{\rho} f\left(x^{*}\right)=\delta^{\rho} g\left(y^{*}\right) \geq g\left(\nu^{*}\right) .
$$

Noting that $\bar{\nu}=\arg \min \left\{g(\nu) \mid \nu \in B^{\delta}\right\}$, we can know

$$
g\left(\nu^{*}\right) \geq g(\bar{\nu}) .
$$

Since $x_{\bar{v}}$ is the optimal solution to the linear programming problem $\left(\mathrm{LP}_{\bar{\gamma}}\right)$, let

$$
\tilde{v}_{i}=f_{i}\left(x_{\bar{v}}\right), \quad i=1,2, \ldots, p .
$$

Apparently, $\widetilde{v}=\left(\widetilde{v}_{1}, \widetilde{v}_{2}, \ldots, \widetilde{v}_{p}\right) \in H$. So, by taking advantage of the formula (16) in Theorem 5, we have

$$
g(\bar{\nu}) \geq g(\widetilde{\nu})=\prod_{i=1}^{p}\left(\widetilde{v}_{i}\right)^{\alpha_{i}}=\prod_{i=1}^{p}\left(f_{i}\left(x_{\bar{\nu}}\right)\right)^{\alpha_{i}}=f\left(x_{\bar{\nu}}\right) .
$$

Therefore, by integrating formulas (35) and (38) and combining the $\delta=(1+\varepsilon)^{(1 / \rho)}$, we can obtain

$$
f\left(x_{\bar{v}}\right) \leq(1+\varepsilon) f\left(x^{*}\right)
$$

and this proof is completed.

Remark 1. According to Theorem 6, if $y^{*} \in B^{\delta}$, then from Theorem 5, the optimal solution $x_{y^{*}}$ of the linear programming problem $\left(\mathrm{LP}_{y^{*}}\right)$ is exactly the global optimal solution of the original problem GLMP. 
Through Theorem 6, we can see that for a given precision $\varepsilon \in(0,1)$, Algorithm 1 will obtain a global $\varepsilon$-approximation solution to the problem GLMP. Moreover, Remark 1 also shows that if $y^{*} \in B^{\delta}$, then Algorithm 1 will find a global optimal solution of the problem GLMP exactly.

3.2. Accelerating Techniques. Algorithm 1 shows that, for any $\nu \in B^{\delta}$, it is required to solve the linear programming problem $\left(\mathrm{LP}_{\nu}\right)$, in order to verify that the $D(\nu)$ is nonempty. Hence, the computational cost of Algorithm 1 depends on the number of points within the set $B^{\delta}$, respectively. Then, the proposal of the acceleration technique will discard some points that are not necessary to consider the set $B^{\delta}$ and only consider the region that contains the global optimal solution of the problem EOP. The detailed process is given below.

If $\bar{v}$ is the best known solution to the problem EOP, $x_{\bar{\gamma}}$ is the optimal solution to the linear programming problem $\left(\mathrm{LP}_{\bar{\nu}}\right) ; \quad$ for each $i=1,2, \ldots, p$, let $\tilde{v}_{i}=f_{i}\left(x_{\bar{\nu}}\right), \widetilde{v}=\left(\widetilde{v}_{1}, \widetilde{v}_{2}, \ldots, \widetilde{v}_{p}\right)^{T} ; \quad$ obviously $g(\widetilde{\nu}) \leq g(\bar{\nu}) ;$ then, $\widetilde{v}$ may be a better solution than $\bar{\nu}$. Well, using $\widetilde{v}$ may be able to remove more vertices from $B^{\delta}$ that do not need to be explored. To give the acceleration technique for Algorithm 1, we first need to specify a necessary condition that the points in each subrectangle $H^{k} \subseteq H^{0}=H(k \geq 1)$ containing the global optimal solution of the problem EOP must be satisfied, that is,

$$
\prod_{i=1}^{p} l_{i}^{\alpha_{i}} \leq g\left(y^{*}\right) \leq g(y) \leq g(\widetilde{v}), \quad \forall y \in H^{k}
$$

where $H^{k}=\left[l, u^{k}\right], \quad u^{k}=\left(u_{1}^{k}, u_{2}^{k}, \ldots, u_{p}^{k}\right)^{T}, \quad u_{i}^{k} \leq u_{i}^{k-1} \leq u_{i}$, $i=1,2, \ldots, p$. Similarly, if $\delta=(1+\varepsilon)^{\left(1 \rho_{\rho}\right)}$ are used to segment rectangles $H^{k}$ on each dimension, this will produce a limited number of small rectangles. For this purpose, let

$$
\gamma_{i}^{k}=\arg \max \left\{\sigma \in \mathbb{N} \mid l_{i} \delta^{\sigma} \leq u_{i}^{k}\right\}, \quad i=1,2, \ldots, p .
$$

Then, a set of vertices of a finite number of small rectangles will also be generated on a rectangular $H^{k}$, that is,

$$
B_{k}^{\delta}=\left\{v_{1}, v_{2}, \ldots, v_{p} \mid v_{i} \in P_{k i}^{\delta}, i=1,2, \ldots, p\right\},
$$

where $P_{k i}^{\delta}=\left\{l_{i}, l_{i} \delta, \ldots, l_{i} \delta^{\gamma_{i}^{k}}\right\}$. Clearly, $B_{k}^{\delta} \subseteq B_{0}^{\delta}=B^{\delta}$ and $B_{k}^{\delta} \subset H^{k} \subseteq H_{0}=H$.

Based on the above discussion, we will give Propositions 1 and 2 to clarify the acceleration techniques of the algorithm.

Proposition 1. The global optimal solution of the problem EOP cannot be obtained on the set $\bar{B}_{k i}^{\delta}$ if a $i \in\{1,2, \ldots, p\}$ makes $\left(g(\widetilde{\nu}) / M_{i}\right)^{\left(1 / \alpha_{i}\right)}<l_{i} \delta^{\gamma_{i}^{k}}$, of which

$$
\bar{B}_{k i}^{\delta}=\left\{\nu \in B_{k}^{\delta} \mid\left(\frac{g(\widetilde{\nu})}{M_{i}}\right)^{\left(1 / \alpha_{i}\right)}<v_{i}\right\}, \quad i \in\{1,2, \ldots, p\} .
$$

Proof. If $v \in \bar{B}_{k i}^{\delta}$, then there must be $\left(g(\widetilde{\nu}) / M_{i}\right)^{\left(1 / \alpha_{i}\right)}<v_{i} \leq l_{i} \delta^{\gamma_{i}^{k}}$, and thus there is

$$
g(\widetilde{\nu})=\left(\left(\frac{g(\widetilde{\nu})}{M_{i}}\right)^{\left(1 / \alpha_{i}\right)}\right)^{\alpha_{i}} M_{i}<\left(\nu_{i}\right)^{\alpha_{i}} M_{i}=\left(v_{i}\right)^{\alpha_{i}} \prod_{j=1, j \neq i}^{p} l_{j}^{\alpha_{j}} \leq \prod_{j=1}^{p}\left(v_{j}\right)^{\alpha_{j}}=g(\nu),
$$

which contradicts the inequality chain (40), so the conclusion is valid.

With Proposition 1, we generate a new rectangle $H^{k+1}$ and vertex set $B_{k+1}^{\delta}$, i.e., for each $i=1,2, \ldots, p$, let

$$
u_{i}^{k+1}= \begin{cases}\left(\frac{g(\widetilde{\nu})}{M_{i}}\right)^{\left(1 / \alpha_{i}\right)}, & \left(\frac{g(\widetilde{\nu})}{M_{i}}\right)^{\left(1 / \alpha_{i}\right)}<l_{i} \delta^{\gamma_{i}^{k}}, \\ u_{i}^{k}, & \left(\frac{g(\widetilde{\nu})}{M_{i}}\right)^{\left(1 / \alpha_{i}\right)} \geq l_{i} \delta^{\gamma_{i}^{k}},\end{cases}
$$

as well as

$$
\gamma_{i}^{k+1}= \begin{cases}\arg \max \left\{\sigma \in \mathbb{N} \mid l_{i} \delta^{\sigma} \leq u_{i}^{k+1}\right\}, & \left(\frac{g(\widetilde{\nu})}{M_{i}}\right)^{\left(1 / \alpha_{i}\right)}<l_{i} \delta^{\gamma_{i}^{k}}, \\ \gamma_{i}^{k}, & \left(\frac{g(\widetilde{\nu})}{M_{i}}\right)^{\left(1 / \alpha_{i}\right)} \geq l_{i} \delta^{\gamma_{i}^{k}} .\end{cases}
$$

Well, $u^{k+1}=\left[l, u^{k+1}\right]$ with $u^{k+1}=\left(u_{1}^{k+1}, u_{2}^{k+1}, \ldots, u_{p}^{k+1}\right)$.

Moreover, the above rules may produce a small rectangular vertex set $B_{k+1}^{\delta}$ with relatively few new elements, but there is still $\widetilde{v} \in B_{k+1}^{\delta}$, so we then give Proposition 2 to delete the other unconsidered elements in $B_{k+1}^{\delta}$.

Proposition 2. If $\bar{v}$ is the best known solution to the problem $E O P, x_{\bar{v}}$ is the optimal solution to the linear programming problem $\left(L P_{\bar{v}}\right) ; \quad$ for each $i=1,2, \ldots, p$, let $\widetilde{v}_{i}=f_{i}\left(x_{\bar{v}}\right), \widetilde{v}=\left(\widetilde{v}_{1}, \widetilde{v}_{2}, \ldots, \widetilde{v}_{p}\right)^{T}$, and define the set

$$
\bar{B}_{k+1}^{\delta}=\left\{\nu \in B_{k+1}^{\delta} \mid \widetilde{v}_{i} \leq v_{i}, i=1,2, \ldots, p\right\} .
$$

Then, for any $v \in \bar{B}_{k+1}^{\delta}$, the EOP cannot get a better solution than $\tilde{\nu}$.

Proof. Since $x_{\bar{v}}$ is the optimal solution to a linear programming problem $\left(\mathrm{LP}_{\bar{\nu}}\right)$, then there is at least one point $x_{\bar{\nu}}$ in the set $D(\widetilde{\nu})$, so $D(\widetilde{\nu}) \neq \varnothing$. For arbitrary $\nu \in \bar{B}_{k+1}^{\delta}$, obviously $D(\widetilde{\nu}) \subseteq D(\nu)$, and thus $D(\nu) \neq \varnothing$. According to the 
definition of the function $g(y)$, for each $v \in \bar{B}_{k+1}^{\delta}$, the objective function value of the EOP meets

$$
g(\nu)=\prod_{i=1}^{p}\left(v_{i}\right)^{\alpha_{i}} \geq \prod_{i=1}^{p}\left(\widetilde{v}_{i}\right)^{\alpha_{i}}=g(\widetilde{v}),
$$

and this conclusion is proved.

Next, for a given $\varepsilon \in(0,1), \delta=(1+\varepsilon)^{(1 / \rho)}$, make use of Proposition 2; let

$$
\tau_{i}^{k+1}=\arg \min \left\{\sigma \in \mathbb{N} \mid \widetilde{v}_{i} \leq l_{i} \delta^{\sigma} \leq u_{i}^{k+1}\right\} .
$$

Through the expression of $\gamma_{i}^{k+1}$ in (46), the set $\bar{B}_{k+1}^{\delta}$ is defined as follows.

$$
\begin{aligned}
\bar{B}_{k+1}^{\delta} & =\left\{l_{i} \delta^{\sigma_{1}}, l_{i} \delta^{\sigma_{2}}, \ldots, l_{i} \delta^{\sigma_{p}} \mid \sigma_{i}\right. \\
& \left.\in\left\{\tau_{i}^{k+1}, \tau_{i}^{k+1}+1, \ldots, \gamma_{i}^{k+1}\right\}, i=1,2, \ldots, p\right\} .
\end{aligned}
$$

Therefore, for the convenience of narration, let $S_{k+1}^{\delta}=B_{k+1}^{\delta} \backslash \bar{B}_{k+1}^{\delta}$. This means that in order to obtain a global $\varepsilon$-approximation solution for problem EOP, it is only necessary to calculate up to $\left|S_{k+1}^{\delta}\right|$ linear programming subproblems $\left(\mathrm{LP}_{\gamma}\right)$ to determine whether the $D(\nu)$ is not empty, which determines the function value $g(v)$ at each vertex $v \in S_{k+1}^{\delta}$. Then, by using the set $S_{k+1}^{\delta}$, the computational efficiency of Algorithm 1 will be improved, leading to the following algorithm.

$$
\begin{aligned}
& \widetilde{v}_{i}=f_{i}\left(x_{\bar{v}}\right), \quad i=1,2, \ldots, p, \\
& \widetilde{v}=\left(\widetilde{v}_{1}, \widetilde{v}_{2}, \ldots, \widetilde{v}_{p}\right)^{T},
\end{aligned}
$$

and then $x_{\bar{v}}, \widetilde{v}$ is a global $\mathcal{E}$-approximation solution to the problems GLMP and EOP, respectively.

Note that the Algorithm 2 simply removes the set of vertices that do not contain a global optimal solution; therefore, it is similar to Theorem 6; Algorithm 2 will also return a global $\varepsilon$-approximation solution of the problem GLMP and EOP as well.

\section{Analysis of Computational Complexity of the Algorithm}

We first give Lemma 1 to discuss the computational complexity of the two algorithms.

Lemma 1 (see [22]). Let $\lambda$ be the maximum of the absolute values of all the elements $A, b, c_{i}, d_{i}$ in problem GLMP; then, each component $x_{j}^{0}$ of any pole $x^{0}$ of $X$ can be expressed as $x_{j}^{0}=\left(p_{j} / q\right)$, where $0 \leq p_{j} \leq(n \lambda)^{n}, \quad 0<q \leq(n \lambda)^{n}$, $j=1,2, \ldots, n$.

Because for each $i=1,2, \ldots, p$, the solution $\tilde{x}^{i}$ to the linear programming problem $l_{i}=\min _{x \in X} f_{i}(x)$ is the pole of $X$, by Lemma 1 , we have $\widetilde{x}_{j}^{i}=\left(p_{j}^{i} / q^{i}\right)$, where $0 \leq p_{j}^{i} \leq(n \lambda)^{n}$, $0<q^{i} \leq(n \lambda)^{n}, \quad j=1,2, \ldots, n . \quad$ Thus, $l_{i}=\sum_{j=1}^{n} c_{i j}\left(p_{j}^{i} / q^{i}\right)+d_{i}, i=1,2, \ldots, p$. Moreover, let

$$
\begin{aligned}
& \widetilde{q}=\max \left\{\frac{1}{q^{i}} \mid i=1,2, \ldots, p\right\}, \\
& \omega=\min \left\{l_{i} \mid i=1,2, \ldots, p\right\}, \\
& \widetilde{U}=f(\check{x})=\min _{1 \leq i \leq p} f\left(\widetilde{x}^{i}\right),
\end{aligned}
$$

and for the sake of the following smooth description of Theorem 7, here $\check{x}$ is defined in Theorem 1 .

Theorem 7. For a given $p \geq 2$, in order to obtain a global $\varepsilon$-approximation solution to the problem GLMP, the upper limit of the time required for the proposed Algorithm 1 is

$$
O\left(\left(\frac{2 \widetilde{\alpha} \rho^{2}}{\varepsilon}[(n+1) \ln (n \lambda)-\ln \omega]+1\right)^{p} \cdot T(m+p, n)\right),
$$

where $\widetilde{\alpha}=\max \left\{\left(1 / \alpha_{i}\right) \mid i=1,2, \ldots, p\right\}, \quad \rho=\sum_{i=1}^{p} \alpha_{i}, \quad$ and $T(m+p, n)$ represents the upper limit of the time used to solve a linear programming problem with $m+p$ linear constraints and $n$ variables at a time.

Proof. From the formulas (22) and (23), we can see that the maximum number of midpoint of the set $B^{\delta}$ is

$$
\prod_{i=1}^{p}\left(\log _{\delta} \frac{u_{i}}{l_{i}}+1\right)
$$

Using the definition of $\tilde{q}, \omega$ in formula (52) and Lemma 1 , we have

$$
\omega \leq l_{i} \leq \widetilde{q} n \lambda(n \lambda)^{n}+\lambda \leq 2 \widetilde{q}(n \lambda)^{n+1}, \quad i=1,2, \ldots, p .
$$

Furthermore, we also have

$$
\widetilde{U}=\prod_{i=1}^{p}\left(c_{i}^{T} \check{x}+d_{i}\right)^{\alpha_{i}} \leq \prod_{i=1}^{p}\left(2 \widetilde{q}(n \lambda)^{n+1}\right)^{\alpha_{i}}=\left(2 \widetilde{q}(n \lambda)^{n+1}\right)^{\sum_{i=1}^{p} \alpha_{i}},
$$

by using formula (53) and the above inequality (56). Of course, according to the definition of $M_{i}$ and $u_{i}$ in Theorem 1 , and in conjunction with $\rho=\sum_{i=1}^{p} \alpha_{i}$, there will be

$$
u_{i}=\left(\frac{\widehat{U}}{M_{i}}\right)^{\left(1 / \alpha_{i}\right)} \leq\left(2 \widetilde{q}(n \lambda)^{n+1}\right)\left(\frac{2 \widetilde{q}(n \lambda)^{n+1}}{\omega}\right)^{\left(\rho / \alpha_{i}\right)-1} .
$$

By means of above formulas (56) and (58), we can have

$$
\frac{u_{i}}{l_{i}} \leq\left(\frac{2 \widetilde{q}(n \lambda)^{n+1}}{\omega}\right)^{\left(\rho / \alpha_{i}\right)},
$$

and thus

$$
\begin{aligned}
\ln \frac{u_{i}}{l_{i}} & \leq \frac{\rho}{\alpha_{i}}[\ln 2 \widetilde{q}+(n+1) \ln (n \lambda)-\ln \omega] \\
& \leq \rho \widetilde{\alpha}[\ln 2 \widetilde{q}+(n+1) \ln (n \lambda)-\ln \omega] .
\end{aligned}
$$


(1) Step 0 (initialization). Set $\varepsilon \in(0,1), \delta=(1+\varepsilon)^{(1 / \rho)}$. By using formulas $(22)$ and $(23), H^{0}=H$ is subdivided into smaller rectangles, such that the ratio of two consecutive segments is $\delta$ in each dimension. Represent the vertex of each small rectangle as $\nu=\left(\nu_{1}, v_{2}, \ldots, v_{p}\right)$, which is stored in the set $B^{\delta}$. Let $F=+\infty, T=\varnothing, B_{0}^{\delta}=B^{\delta}, \Xi^{0}=B_{0}^{\delta}, k=0$.

(2) Step 1. Select a point $\nu=\left(\nu_{1}, v_{2}, \ldots, v_{p}\right)^{T}$ from the $\Xi^{k}$, solve the linear programming problem $\left(\mathrm{LP}_{\nu}\right)$, and let $T=T \cup \nu$.

(3) Step 2. If the problem $\left(\mathrm{LP}_{v}\right)$ is solvable, then $D(\nu) \neq \varnothing$, and let $g(\nu)=\prod_{i=1}^{p}\left(\nu_{i}\right)^{\alpha_{i}}$; if $g(\nu)<F$, let $\bar{v}=\nu, x_{\bar{v}}=x_{\nu}$, $\widetilde{v}=\left(\widetilde{\nu}_{1}, \widetilde{v}_{2}, \ldots, \widetilde{v}_{p}\right)^{T}=\left(f_{1}\left(x_{\bar{\nu}}\right), f_{2}\left(x_{\bar{\gamma}}\right), \ldots, f_{p}\left(x_{\bar{\nu}}\right)\right)^{T}, F=g(\widetilde{\nu})$. Use rules (45) and (46) to produce $H^{k+1}$ and $B_{k+1}^{\delta}$ and use formulas (49) and (50) to obtain set $\bar{B}_{k+1}^{\delta}$; let $S_{k+1}^{\delta}=B_{k+1}^{\delta} \backslash \bar{B}_{k+1}^{\delta}, \Xi^{k}=S_{k+1}^{\delta} \backslash T$. If $\Xi^{k} \neq \varnothing$, set $k=k+1$ and go to Step 1; otherwise, the algorithm terminates; let

Algorithm 2: Improved algorithm.

Using $\varepsilon \in(0,1), \delta=(1+\varepsilon)^{(1 / \rho)}$ in Algorithm 1 and $(\varepsilon / 2)<\ln (1+\varepsilon)<\varepsilon$, then there will be

$$
\log _{\delta} \frac{u_{i}}{l_{i}}=\rho \log (1+\varepsilon) \frac{u_{i}}{l_{i}}=\rho \frac{\ln \left(u_{i} / l_{i}\right)}{\ln (1+\varepsilon)}<\frac{2 \rho \ln \left(u_{i} / l_{i}\right)}{\varepsilon} .
$$

Then, by using the above formulas (55), (60), and (61), the upper limit of the number (expressed in $\left|B^{\delta}\right|$ ) of interior points of $B^{\delta}$ is

$$
\left|B^{\delta}\right| \leq\left(\frac{2 \widetilde{\alpha} \rho^{2}}{\varepsilon}[\ln 2 \widetilde{q}+(n+1) \ln (n \lambda)-\ln \omega]+1\right)^{p},
$$

in the utilized formula (55), (60), (61). From the above formula (62), we can see that the running time of Algorithm 1 is at most

$$
O\left(\left(\frac{2 \widetilde{\alpha} \rho^{2}}{\varepsilon}[(n+1) \ln (n \lambda)-\ln \omega]+1\right)^{p} \cdot T(m+p, n)\right)
$$

when the global $\varepsilon$-approximation solution is obtained, and then the proof of the conclusion is completed.
Remark 2. Propositions 1 and 2 show that we can accelerate Algorithm 1 by removing the vertices of the small rectangle that needs not be considered, which leads to Algorithm 2 that is more resource-efficient than Algorithm 1; in other words, Algorithm 2 is an improvement on Algorithm 1. Then, the upper bound of the CPU running time required by Algorithm 2 is the same as that of Algorithm 1 in the most extreme cases (where acceleration techniques always fail). Therefore, Algorithm 2 is likewise a polynomial time approximation algorithm.

\section{Numerical Experiments}

This section will test the performance of the algorithm through several test problems. All of our testing procedures were performed via MATLAB (2012a) on computers with Intel(R) Core(TM)i5-2320, $3.00 \mathrm{GHz}$ power processor, 4.00 GB memory, and Microsoft Win7 operating system.

Problem 1 (see $[17,18])$

$$
\begin{aligned}
& \min \begin{array}{l}
\left(0.813396 x_{1}+0.67440 x_{2}+0.305038 x_{3}+0.129742 x_{4}+0.217796\right) \\
\times\left(0.224508 x_{1}+0.063458 x_{2}+0.932230 x_{3}+0.528736 x_{4}+0.091947\right) \\
-0.324014 x_{1}-0.501754 x_{2}-0.719204 x_{3}+0.099562 x_{4} \leq-0.052215, \\
0.445225 x_{1}-0.346896 x_{2}+0.637939 x_{3}-0.257623 x_{4} \leq 0.427920, \\
-0.202821 x_{1}+0.647361 x_{2}+0.920135 x_{3}-0.983091 x_{4} \leq 0.840950, \\
-0.886420 x_{1}-0.802444 x_{2}-0.305441 x_{3}-0.180123 x_{4} \leq-1.353686, \\
-0.515399 x_{1}-0.424820 x_{2}+0.897498 x_{3}+0.187268 x_{4} \leq 2.137251, \\
-0.591515 x_{1}+0.060581 x_{2}-0.427365 x_{3}+0.579388 x_{4} \leq-0.290987, \\
0.423524 x_{1}+0.940496 x_{2}-0.437944 x_{3}-0.742941 x_{4} \leq 0.373620, \\
x_{1} \geq 0, x_{2} \geq 0, x_{3} \geq 0, x_{4} \geq 0 .
\end{array} \\
& \text { s.t. }
\end{aligned}
$$


Problem 2 (see $[17,18])$

$$
\begin{aligned}
& \min \left(3 x_{1}-2 x_{2}-2\right)^{(2 / 3)}\left(x_{1}+2 x_{2}+2\right)^{(2 / 5)} \\
& \text { s.t. }\left\{\begin{array}{l}
2 x_{1}-x_{2} \geq 2, x_{1}-2 x_{2} \leq 2, \\
x_{1}+x_{2} \leq 5,3 \leq x_{1} \leq 5,1 \leq x_{2} \leq 3 .
\end{array}\right.
\end{aligned}
$$

Problem 3 (see $[8,17,18])$

$$
\begin{aligned}
& \min \left(x_{1}+x_{2}+1\right)^{2.5}\left(2 x_{1}+x_{2}+1\right)^{1.1}\left(x_{1}+2 x_{2}+1\right)^{1.9} \\
& \text { s.t. }\left\{\begin{array}{l}
x_{1}+2 x_{2} \leq 6 \\
2 x_{1}+x_{2} \leq 8 \\
1 \leq x_{1} \leq 3 \\
1 \leq x_{2} \leq 3
\end{array}\right.
\end{aligned}
$$

Problem 4 (see [20])

$$
\begin{aligned}
& \min \left(x_{1}+x_{2}\right)\left(x_{1}-x_{2}+7\right) \\
& \text { s.t. }\left\{\begin{array}{l}
2 x_{1}+x_{2} \leq 14, \\
x_{1}+x_{2} \leq 10, \\
-4 x_{1}+x_{2} \leq 0, \\
2 x_{1}+x_{2} \geq 6, \\
x_{1}+2 x_{2} \geq 6, \\
x_{1}-x_{2} \leq 3, \\
1.99 \leq x_{1} \leq 2.01, \\
7.99 \leq x_{2} \leq 8.01 .
\end{array}\right.
\end{aligned}
$$

Problem 5 (see [19])

$$
\begin{aligned}
& \min \left(c_{1}^{T} x+d_{1}\right)\left(c_{2}^{T} x+d_{2}\right) \\
& \text { s.t. } A x=b, x \geq 0,
\end{aligned}
$$

where

$$
\begin{aligned}
& A=\left(\begin{array}{ccccccccccc}
9 & 9 & 2 & 1 & 0 & 0 & 0 & 0 & 0 & 0 & 0 \\
8 & 1 & 8 & 0 & 1 & 0 & 0 & 0 & 0 & 0 & 0 \\
1 & 8 & 8 & 0 & 1 & 0 & 0 & 0 & 0 & 0 & 0 \\
7 & 1 & 1 & 0 & 0 & 0 & -1 & 0 & 0 & 0 & 0 \\
1 & 7 & 1 & 0 & 0 & 0 & 0 & -1 & 0 & 0 & 0 \\
1 & 1 & 7 & 0 & 0 & 0 & 0 & 0 & -1 & 0 & 0 \\
1 & 0 & 0 & 0 & 0 & 0 & 0 & 0 & 0 & 1 & 0 \\
0 & 1 & 0 & 0 & 0 & 0 & 0 & 0 & 0 & 0 & 1
\end{array}\right) \\
& b=(81,72,72,9,9,9,8,8)^{T} \text {, } \\
& c_{1}=\left(1,0, \frac{1}{9}, 0,0,0,0,0,0,0,0\right)^{T} \text {, } \\
& c_{2}=\left(0,1, \frac{1}{9}, 0,0,0,0,0,0,0,0\right)^{T} \text {, } \\
& d_{1}=0 \text {, } \\
& d_{2}=0 \text {. }
\end{aligned}
$$

Obviously, Problem 5 can be transformed into the following forms:

$$
\min \left(x_{1}+\frac{1}{9} x_{3}\right)\left(x_{2}+\frac{1}{9} x_{3}\right)
$$

$$
\text { s.t. }\left\{\begin{array}{l}
9 x_{1}+9 x_{2}+2 x_{3} \leq 81, \\
8 x_{1}+x_{2}+8 x_{3} \leq 72, \\
x_{1}+8 x_{2}+8 x_{3} \leq 72, \\
7 x_{1}+x_{2}+x_{3} \geq 9, \\
x_{1}+7 x_{2}+x_{3} \geq 9 \\
x_{1}+x_{2}+7 x_{3} \geq 9 \\
0 \leq x_{1} \leq 8 \\
0 \leq x_{2} \leq 8 \\
0 \leq x_{3} \leq 9
\end{array}\right.
$$

Problem 6 (see [8])

$$
\begin{aligned}
& \min \left(3 x_{1}-4 x_{2}+5\right)\left(x_{1}+2 x_{2}-1\right)^{0.5}\left(2 x_{1}-x_{2}+4\right) \\
& \times\left(x_{1}-2 x_{2}+8\right)^{0.5}\left(2 x_{1}+x_{2}-1\right) \\
& \text { s.t. }\left\{\begin{array}{l}
5 x_{1}-8 x_{2} \geq-24, \\
5 x_{1}+8 x_{2} \leq 44, \\
6 x_{1}-3 x_{2} \leq 15, \\
4 x_{1}+5 x_{2} \geq 10, \\
1 \leq x_{1} \leq 3 \\
0 \leq x_{2} \leq 1 .
\end{array}\right.
\end{aligned}
$$

Problem 7

$$
\min \prod_{i=1}^{p}\left(c_{i}^{T} x_{j}+d_{i}\right)^{\alpha_{i}} \text { s.t. } \quad A x \leq b, x \geq 0,
$$

where $p \geq 2, \quad c_{i} \in \mathbb{R}^{n}(i=1,2, \ldots, p)$ are pseudo-random numbers in $[0,1], \alpha_{i}(i=1,2, \ldots, p)$ are pseudo-random numbers in [0.00001, 1], $d_{i}=1$, constraint matrix elements $a_{i j}$ are generated in $[-1,1]$ via $a_{i j}=2 * \emptyset-1$, in which $₫$ are pseudo-random numbers in $[0,1]$, and the right-hand side values are generated via $b_{i}=\sum_{j=1}^{n} a_{i j}+2 \beta_{i}$, in which $\beta_{i}$ are pseudo-random numbers in $[0,1]$.

The numerical results in Tables 1 and 2 show that Algorithms 1 and 2 can effectively solve the three test problems known in the literature and get an approximate solution, so both algorithms are feasible.

Further, we do the corresponding random numerical experiments through Problem 7, which is utilized to explore the performance of the two algorithms. We determine the convergence accuracy of the algorithm to 0.05 . For each set 
Table 1: Comparison of results in Problems 1-6.

\begin{tabular}{|c|c|c|c|}
\hline Problem & Reference & Optimal solution & Optimal optimum \\
\hline 1 & $\begin{array}{c}\text { Locatelli [17] } \\
\text { Shen and Wang [18] } \\
\text { Liu and Zhao [8] } \\
\text { Algorithms } 1 / 2\end{array}$ & $\begin{array}{c}(1.3148,0.1396,0.0000,0.4233) \\
(1.3148,0.1396,0.0000,0.4233) \\
\left(1.3148,0.13955,2.6891 \times 10^{-14}, 0.42329\right) \\
(1.3148,0.1396,0.0000,0.4233)\end{array}$ & $\begin{array}{l}0.890190 \\
0.890190 \\
0.890190 \\
0.890190\end{array}$ \\
\hline 2 & $\begin{array}{c}\text { Locatelli [17] } \\
\text { Shen and Wang [18] } \\
\text { Liu and Zhao [8] } \\
\text { Algorithm 1 } \\
\text { Algorithm 2 }\end{array}$ & $\begin{array}{l}(3.000,2.000) \\
(3.000,2.000) \\
(3.000,2.000) \\
(3.000,2.000) \\
(3.000,2.000) \\
\end{array}$ & $\begin{array}{l}5.014514 \\
5.009309 \\
5.009309 \\
5.009309 \\
5.009309 \\
\end{array}$ \\
\hline 3 & $\begin{array}{c}\text { Liu and Zhao [8] } \\
\text { Locatelli [17] } \\
\text { Shen and Wang [18] } \\
\text { Algorithm } 1 / 2\end{array}$ & $\begin{array}{l}(1,1) \\
(1,1) \\
(1,1) \\
(1,1)\end{array}$ & $\begin{array}{l}997.661265 \\
997.661265 \\
997.661265 \\
997.661265 \\
\end{array}$ \\
\hline 4 & $\begin{array}{c}\text { Shen and Hang [20] } \\
\text { Algorithm 1/2 }\end{array}$ & $\begin{array}{l}(2,8) \\
(2,8) \\
\end{array}$ & $\begin{array}{l}10 \\
10 \\
\end{array}$ \\
\hline 5 & $\begin{array}{l}\text { Zhang et al. [19] } \\
\text { Algorithm } 1 \\
\text { Algorithm } 2 \\
\end{array}$ & $\begin{array}{l}(0.0,8.0,1.0, \ldots) \\
(0.0,8.0,1.0, \ldots) \\
(0.0,8.0,1.0, \ldots) \\
\end{array}$ & $\begin{array}{l}0.91235 \\
0.91235 \\
0.91235 \\
\end{array}$ \\
\hline 6 & $\begin{array}{l}\text { Liu and Zhao [8] } \\
\text { Algorithm } 1 \\
\text { Algorithm } 2\end{array}$ & $\begin{array}{l}(1.25,1) \\
(1.25,1) \\
(1.25,1)\end{array}$ & $\begin{array}{l}263.785989 \\
263.785989 \\
263.785989\end{array}$ \\
\hline
\end{tabular}

Table 2: Comparison of results in Problems 1-6.

\begin{tabular}{|c|c|c|c|c|}
\hline Problem & Reference & Iter & Time & $\varepsilon$ \\
\hline \multirow{3}{*}{1} & Locatelli [17] & 404 & 9.606 & 0.05 \\
\hline & Shen and Wang [18] & 3 & 0.047 & 0.05 \\
\hline & Algorithm $1 / 2$ & 1 & 0.0149 & 0.05 \\
\hline \multirow{4}{*}{2} & Locatelli [17] & 69 & 2.4960 & 0.15009 \\
\hline & Shen and Wang [18] & 4 & 0.0800 & 0.15009 \\
\hline & Algorithm 1 & 6 & 0.1024 & 0.15009 \\
\hline & Algorithm 2 & 4 & 0.0657 & 0.15009 \\
\hline \multirow{3}{*}{3} & Locatelli [17] & 5 & 1.126 & 0.2 \\
\hline & Shen and Wang [18] & 4 & 0.085 & 0.2 \\
\hline & Algorithm $1 / 2$ & 1 & 0.0116 & 0.2 \\
\hline 4 & Algorithm 1/2 & 1 & 0.0241 & 0.01 \\
\hline \multirow{2}{*}{5} & Algorithm 1 & 797 & 47.5367 & 0.2 \\
\hline & Algorithm 2 & 507 & 30.2111 & 0.2 \\
\hline \multirow{2}{*}{6} & Algorithm 1 & 63 & 59.4304 & 0.2 \\
\hline & Algorithm 2 & 37 & 35.6072 & 0.2 \\
\hline
\end{tabular}

of fixed parameters $(p, m, n)$, we run the two algorithms 10 times for numerical comparison, and the numerical results are given in Table 3. In Table 3, Avg (Std) time and Avg (Std) Iter represent the average (standard deviation) of the CPU running time and the average (standard deviation) of iterations, respectively, after the algorithm has run 10 times. Table 3 shows that the computation effect of Algorithm 2 is better than that of Algorithm 1, mainly because our acceleration technique plays a significant role by deleting the vertices of small rectangles that do not need to be considered. Hence, we believe that this acceleration technique may be generalized on other approximation algorithms such as $[17,18,20]$.

Moreover, under the condition that the fixed parameters $(p, m)$ are invariant, the CPU running time of the two algorithms will increase with the scale $n$ of Problem 7. Under the condition that the prefixed parameters $(m, n)$ are invariant, the CPU running time and iterations of the two 
Table 3: Comparison of numerical results by using Problem 7.

\begin{tabular}{|c|c|c|c|c|}
\hline \multirow{2}{*}{$(p, m, n)$} & \multicolumn{2}{|c|}{ Algorithm 1} & \multicolumn{2}{|c|}{ Algorithm 2} \\
\hline & Avg (Std) time & Avg (Std) Iter & Avg (Std) time & Avg (Std) Iter \\
\hline$(2,10,20)$ & $2.9068(2.8062)$ & $75.8(84.7700)$ & $1.9686(1.9352)$ & $22.5(27.3395)$ \\
\hline$(2,20,20)$ & $2.3784(3.1017)$ & $52.6(76.8936)$ & $1.7129(2.1472)$ & $23.4(35.1801)$ \\
\hline$(2,22,20)$ & $0.8663(0.9232)$ & $18.1(25.0257)$ & $0.6568(0.6239)$ & $8(10.0199)$ \\
\hline$(2,20,30)$ & $6.2414(6.3274)$ & $165.2(164.0334)$ & $3.4923(3.7124)$ & $49(61.764)$ \\
\hline$(2,35,50)$ & $3.9868(4.4041)$ & $66.4(78.2102)$ & $3.3046(3.9017)$ & $32.3(38.6886)$ \\
\hline$(2,45,60)$ & $5.8908(5.4016)$ & $129.1(125.2481)$ & $3.7409(3.3526)$ & $40.5(38.1084)$ \\
\hline$(2,45,100)$ & $6.6579(5.9685)$ & $125.3(123.7061)$ & $4.2665(3.6485)$ & $40.2(40.1343)$ \\
\hline$(2,60,100)$ & $7.8626(6.3057)$ & $96.6(99.4818)$ & $4.5517(2.8324)$ & $26(19.8343)$ \\
\hline$(2,70,100)$ & $9.1245(8.1057)$ & $96.3(104.6633)$ & $5.0942(3.3528)$ & $23.6(18.9430)$ \\
\hline$(2,70,120)$ & $11.2742(13.2311)$ & $148(215.2185)$ & $6.0341(5.5968)$ & $35(37.3256)$ \\
\hline$(2,100,10)$ & $0.1877(0.1300)$ & $2.4(2.9732)$ & $0.1542(0.0663)$ & $1.3(0.6403)$ \\
\hline$(2,100,50)$ & $0.9029(0.5392)$ & $8.9(7.0632)$ & $0.6542(0.3654)$ & $3.9(2.7730)$ \\
\hline$(2,100,100)$ & $9.8811(8.0793)$ & $68.6(55.5287)$ & $6.9462(6.3403)$ & $24.1(26.6813)$ \\
\hline$(2,100,150)$ & $15.4331(10.2573)$ & $97.4(75.1720)$ & $9.8838(6.2545)$ & $30.8(22.1260)$ \\
\hline$(2,100,200)$ & $27.1157(25.3267)$ & $124.4(130.8076)$ & $18.9561(16.8612)$ & $49.2(47.3810)$ \\
\hline$(2,100,250)$ & $64.8144(72.0125)$ & $285.1(353.7955)$ & $40.3711(41.0487)$ & $91(103.9576)$ \\
\hline$(2,100,300)$ & $87.5572(100.4846)$ & 331 (398.8197) & $55.5067(64.5147)$ & $117.2(153.2434)$ \\
\hline$(2,100,400)$ & $132.4251(176.2381)$ & $363.9(581.9823)$ & $87.0321(97.4482)$ & 130.7 (169.6585) \\
\hline$(2,100,500)$ & $158.4767(183.7060)$ & $338.3(493.9785)$ & $111.0958(106.7086)$ & $133.8(145.3470)$ \\
\hline$(2,100,700)$ & $331.3275(351.8534)$ & $414.2(546.8741)$ & $272.7311(264.9189)$ & $227.1(257.8927)$ \\
\hline$(2,100,1000)$ & $1020.9318(880.7910)$ & $1063.6(1019.7921)$ & $778.8913(638.1782)$ & $522(460.2479)$ \\
\hline$(3,100,10)$ & $4.4724(7.4341)$ & $59.7(117.2502)$ & $3.6522(5.7934)$ & $35.2(55.7867)$ \\
\hline$(3,100,50)$ & $90.8139(74.9843)$ & $1062.4(982.8277)$ & $57.8342(55.5978)$ & $473.3(564.6533)$ \\
\hline$(4,100,10)$ & $75.4301(189.1250)$ & $1509.3(4122.7180)$ & $52.9122(122.0553)$ & $868.1(2203.3283)$ \\
\hline
\end{tabular}

algorithms will grow with the number $(p)$ of linear functions in the objective function of Problem 7.

\section{Concluding Remarks}

In this paper, we mainly propose two polynomial time approximation algorithms that can be utilized to solve the problem GLMP globally, where Algorithm 2 is obtained by accelerating Algorithm 1 by the proposed acceleration technique. The numerical results show that both algorithms are effective and feasible, but the overall calculation effect of Algorithm 2 is better than that of Algorithm 1, which shows that our acceleration technique is efficient and may be extended to some approximation algorithms such as $[17,18,20]$.

\section{Data Availability}

All data and models generated or used during the study are described in Section 5 of this article.

\section{Conflicts of Interest}

The authors declare that they have no conflicts of interest.

\section{Acknowledgments}

This research was supported by the National Natural Science Foundation of China (grant no. 11961001), the Construction Project of First-Class Subjects in Ningxia Higher Education (NXYLXK2017B09), and the Major Proprietary Funded Project of North Minzu University (ZDZX201901).

\section{References}

[1] T. Matsui, "NP-Hardness of linear multiplicative programming and related problems," Journal of Global Optimization, vol. 9, no. 2, pp. 113-119, 1996.

[2] C. Maranas, I. Androulakis, C. Floudas et al., "Solving longterm financial planning problems via global optimization," Journal of Economic Dynamics and Control, vol. 21, no. 8-9, pp. 1405-1425, 1997.

[3] H. Konno, T. Kuno, and Y. Yajima, "Global minimization of a generalized convex multiplicative function," Journal of Global Optimization, vol. 4, no. 1, pp. 47-62, 1994.

[4] J. M. Mulvey, R. J. Vanderbei, and S. A. Zenios, "Robust optimization of large-scale systems," Operations Research, vol. 43, no. 2, pp. 264-281, 1995.

[5] R. Nicholas, P. Layard, and A. Walters, "Microeconomic theory," Economica, vol. 47, no. 186, p. 211, 1980.

[6] H. P. Benson, "Vector maximization with two objective functions," Journal of Optimization Theory and Applications, vol. 28, no. 2, pp. 253-257, 1979.

[7] D. Dennis, "Analyzing public inputs to multiple objective decisions on national forests using conjoint analysis," Forest Ence, vol. 44, no. 3, pp. 421-429, 1998.

[8] S. Liu and Y. Zhao, "An efficient algorithm for globally solving generalized linear multiplicative programming," Journal of Computational and Applied Mathematics, vol. 296, pp. 840847, 2016.

[9] X. J. Liu, T. Umegaki, and Y. Yamamoto, "Heuristic methods for linear multiplicative programming," Journal of Global Optimization, vol. 15, no. 4, pp. 433-447, 1999.

[10] H. P. Benson and G. M. Boger, "Multiplicative programming problems: analysis and efficient point search heuristic," Journal of Optimization Theory and Applications, vol. 94, no. 2, pp. 487-510, 1997. 
[11] P. Shen, X. Bai, and W. Li, "A new accelerating method for globally solving a class of nonconvex programming problems," Nonlinear Analysis: Theory, Methods \& Applications, vol. 71, no. 7-8, pp. 2866-2876, 2009.

[12] Y. Chen and H. Jiao, "A nonisolated optimal solution of general linear multiplicative programming problems," Computers \& Operations Research, vol. 36, no. 9, pp. 25732579, 2009.

[13] C.-F. Wang, Y.-Q. Bai, and P.-P. Shen, "A practicable branchand-bound algorithm for globally solving linear multiplicative programming," Optimization, vol. 66, no. 3, pp. 397-405, 2017.

[14] Y. Gao, C. Xu, and Y. Yang, "An outcome-space finite algorithm for solving linear multiplicative programming," Applied Mathematics and Computation, vol. 179, no. 2, pp. 494-505, 2006

[15] H. Konno and T. Kuno, "Linear multiplicative programming," Mathematical Programming, vol. 56, no. 1-3, pp. 51-64, 1992.

[16] D. Depetrini and M. Locatelli, “A FPTAS for a class of linear multiplicative problems," Computational Optimization and Applications, vol. 44, no. 2, pp. 275-288, 2007.

[17] M. Locatelli, "Approximation algorithm for a class of global optimization problems," Journal of Global Optimization, vol. 55, no. 1, pp. 13-25, 2013.

[18] P. P. Shen and L. F. Wang, "A fully polynomial time approximation algorithm for generalized linear multiplicative programming," Mathematica Applicata, vol. 31, no. 1, pp. 208-213, 2018.

[19] B. Zhang, Y. Gao, X. Liu, and X. Huang, "Output-space branch-and-bound reduction algorithm for a class of linear multiplicative programs," Mathematics, vol. 8 , no. 3, p. 315, 2020.

[20] P. Shen, B. Huang, and L. Wang, "Range division and linearization algorithm for a class of linear ratios optimization problems," Journal of Computational and Applied Mathematics, vol. 350, pp. 324-342, 2019.

[21] C.-F. Wang and S.-Y. Liu, "A new linearization method for generalized linear multiplicative programming," Computers \& Operations Research, vol. 38, no. 7, pp. 1008-1013, 2011.

[22] P. P. Shen and X. K. Zhao, "A polynomial time approximation algorithm for linear fractionl programs," Mathematica Applicata, vol. 26, no. 2, pp. 355-359, 2011. 